\title{
A invenção dos pártons
}

\author{
The invention of pártons
}

\author{
Carlos Ourivio Escobar*1@
}

${ }^{1}$ Fermi National Accelerator Laboratory, Batavia, Illinois, USA

Recebido em 22 de Maio de 2018. Revisado em 23 de Maio de 2018. Aceito em 23 de Maio de 2018

\begin{abstract}
A invenção do modelo a pártons foi uma das maiores contribuições de Richard Feynman à moderna física de partículas. O modelo a pártons surge num momento crítico que é caracterizado pela polarização da física de partículas em duas escolas de pensamento, por um lado a assim chamada democracia nuclear que postulava serem todos hádrons igualmente fundamentais e por outro lado a que começava a formar-se ao redor dos recém propostos quarks e sua utilização, num primeiro momento não como constituintes fundamentais dos hádrons mas sim como entidades matemáticas expressando simetrias de uma teoria quântica de campos (TQC) subjacente, embora ainda desconhecida para as interações fortes. O modelo a pártons transforma-se em pouco tempo numa ferramenta fundamental para a construção desta TQC, chamada Cromodinâmica Quântica, ajudando a promover os quarks de entidades matemáticas a constituintes fundamentais dos hádrons.
\end{abstract}

Palavras-chave: Pártons; Democracia Nuclear; Álgebra de Correntes; Quarks; Glúons

The invention of partons by Richard Feynman was one of his most important contributions to modern particle physics. The parton model appears at a critical moment characterized by the polarization of particle physics in two opposing schools of thought: on the one hand, the so called nuclear democracy, postulating that all hadrons are equally fundamental and on the other hand a school of thought that was starting to form around the idea of quarks and their use at first, not as fundamental constituents of hadrons, but rather as mathematical entities reflecting the internal symmetries of an underlying quantum field theory (QFT) for the strong interaction, at that point still unknown. The parton model rapidly became an important tool in the construction of this QFT, named Quantum Chromodynamics, helping to promote quarks from mathematical entities to fundamental constituents of the hadrons.

Keywords: Partons; Nuclear Democracy; Current Algebra; Quarks; Gluons

\section{Introdução}

Em que trabalhava Richard Feynman no início da década de 1960? A resposta a esta pergunta poderá surpreender a quem leia este artigo. Feynman após seus trabalhos em mecânica quântica (integral de caminhos - ver as contribuições, neste volume, de Alfredo O. Almeida e J. David M. Vianna) e eletrodinâmica quântica (ver os artigos de Arlene Aguiar, Vicente Pleitez e Marcelo Novaes, neste volume) faz incursões na física da matéria condensada (ver os artigos de Nelson Studart e Paulo Farinas) e encerra a década de 1950 com sua seminal contribuição, em coautoria com Murray Gell-Mann, sobre a teoria V-A das interações fracas [1]. Feynman passa então a interessarse pela interação gravitacional, ministrando um curso de pós-graduação no Caltech durante o ano acadêmico 1962-63 e participando de conferências internacionais sobre gravitação [2]. Seu interesse pela gravitação é subitamente interrompido e ele não oculta sua decepção com

*Endereço de correspondência: escobar@fnal.gov o estado das pesquisas nesta área ${ }^{1}$ Aparte seu profundo interesse em ensinar física para estudantes de graduação (ver as contribuições neste volume sobre Feynman e o Ensino de Física) Feynman passa a interessar-se pelas interações fortes, afinal esta era a única das interações fundamentais que ele ainda não havia investigado em profundidade ${ }^{2}$

Para entender que abordagem Feynman usará para estudar a interação hadrônica faremos, na próxima secção, um curto parêntese para descrever brevemente quais eram as abordagens teóricas usadas para estudar os hádrons quando da entrada em cena de Feynman.

\footnotetext{
1 Em uma carta para sua esposa, escrita quando estava numa conferência sobre gravitação em Varsóvia em 1962, ele não oculta sua insatisfação dizendo-lhe que "não estou obtendo nada deste encontro, aprendendo nada...Faça-me lembrar de não vir mais a nenhuma conferência sobre gravitação." [2]

2 Até então seu único trabalho envolvendo hádrons havia sido uma tentativa de explicar a diferença de massa entre o próton e nêutron como sendo de origem eletromagnética [3], o que hoje sabemos não ser inteiramente correto.
} 


\section{Matriz-S e democracia nuclear versus quarks e álgebra de correntes}

Após o extraordinário sucesso obtido com a renormalização da eletrodinâmica quântica, permitindo o cálculo perturbativo em ordens superiores da constante de acoplamento eletromagnético (constante de estrutura fina) e resultados altamente precisos para grandezas como o momento magnético anômalo do elétron e o deslocamento Lamb no átomo de hidrogênio, segue-se um período de grande frustração na aplicação da teoria quântica de campos aos fenômenos hadrônicos devido à impossibilidade de desenvolver uma abordagem perturbativa graças ao valor da constante de interação forte (análoga à constante de estrutura fina da eletrodinâmica quântica) ser muito maior que a unidade. Outra fonte de frustração para os que ainda tentavam usar uma TQC para descrever as interações fortes era a dificuldade em definir quais seriam os campos fundamentais, ou seja, quais entre os hádrons seriam mais fundamentais que os outros? Uma primeira tentativa (Fermi e Yang em 1949) colocava o núcleon (próton e nêutron) como fundamental, ideia depois generalizada por Sakata em 1956 incluindo a estranheza ao promover o bárion estranho lambda à condição de partícula fundamental. A proliferação de hádrons descobertos em raios cósmicos e em aceleradores de partículas no pós-guerra levou à realização que entre os hádrons nenhum deveria ser mais fundamental que outro qualquer. Esta visão foi materializada em uma abordagem teórica conhecida como "democracia nuclear" (Chew e Frautschi, 1961 [4]) usando o formalismo da matriz de espalhamento (Matriz-S), numa tentativa de realizar na física de partículas o que Heisenberg havia conseguido junto com Born e Jordan na formulação da mecânica quântica, conhecida como mecânica matricial que tem como elementos centrais as relações de comutação entre observáveis e os valores esperados destes observáveis. Os defensores da "democracia nuclear" empreenderam uma sistemática oposição aos teóricos de campos quantizados (ver, para uma excelente descrição da atmosfera desta época, o artigo de H. Pietschmann [5]) ao mesmo tempo que alguns dos expoentes em TQC embarcavam em um novo programa que, abrindo mão da descrição dos hádrons em termos de campos fundamentais, fazia uso de elementos de matriz de correntes fracas e eletromagnéticas entre estados hadrônicos, explorando simetrias e comutadores destas correntes (outra vez o programa de Heisenberg!). Um dos praticantes de TQC mais notáveis que embarca nesta nova linha de investigação é Murray Gell-Mann [6] que dois anos depois propõe os quark $\$^{3}$ uma entidade possuindo os números quânticos associados à representa-

\footnotetext{
3 Independentemente George Zweig [8] propõe o que ele chama aces com os mesmos números quânticos que os quarks de Gell-Mann [7], incluindo sua carga elétrica fracionária (1/3 e 2/3 da carga do elétron). Ambos autores enfatizam a necessidade de buscar partículas livres com cargas elétricas fracionárias, um desafio que será levado a serio por vários grupos experimentais e que terá prominência no modelo a pártons.
}

ção fundamental do grupo de simetria $\mathrm{SU}(3)$, com o qual Gell-Mann e independentemente Ne'eman conseguiram uma classificação de todo o espectro hadrônico.

É neste cenário que surge Feynman e seu modelo a pártons, como veremos na próxima seção.

\section{O Modelo a Pártons}

Começamos esta seção com uma pergunta simples: onde pode ser encontrado um artigo de Feynman no qual ele apresenta o seu modelo a pártons no contexto em que este modelo é mais utilizado, ou seja, na análise dos experimentos de espalhamento inelástico profundo léptonnúcleon? A resposta é de certa forma surpreendente já que as ideias e conceitos resumidos na expressão "modelo a pártons" aparecem em um artigo de pouco mais de duas páginas no Physical Review Letters [9], artigo este que é um resumo de sua contribuição bastante longa para uma conferência sobre colisões hadrônicas a altas energias em setembro de 1969. Não há menção em nenhum destes dois trabalhos aos experimentos de espalhamento inelástico profundo lépton-núcleon realizados naquele momento e no ano anterior, 1968, no Stanford Linear Accelerator Center (SLAC). A situação é todavia mais surpreendente se considerarmos que há vários depoimentos comentando a breve visita que Feynman fez ao SLAC em agosto de 1968 (ver, por exemplo Riordan [11], Friedman [12], Close [13]). Nesta visita Feynman encontra-se com alguns membros do grupo experimental que realizava os experimentos de espalhamento inelástico profundo elétron-núcleon e estes mostram a Feynman os primeiros resultados experimentais, com a seção de choque parametrizada da maneira como lhes havia sido sugerida pelo jovem físico teórico de Stanford, James D. Bjorken. Bjorken havia recentemente investigado o espalhamento inelástico profundo usando a álgebra de correntes, e ao examinar o limite em que o momento e energia transferidos pelo elétron ao núcleon vão para o infinito, mantendo sua razão fixa, mostrou que a seção de choque apresenta um comportamento simples no qual as chamadas funções de estrutura (análogas aos mais familiares fatores de forma do espalhamento elástico) são funções apenas de uma variável adimensional, posteriormente notada por $\mathrm{x}_{\mathrm{Bj}}{ }^{4}$ Esta predição de Bjorken, denominada comportamento de escala, foi rapidamente interpretada por Feynman, segundo os depoimentos mencionados acima, como indicando a presença de constituintes elementares dotados de carga elétrica dentro dos núcleons, os pártons de Feynman (ver Close [13] para uma vívida descrição deste episódio).

\footnotetext{
${ }^{4}$ A análise feita por Bjorken do espalhamento inelástico profundo usando algebra de correntes é uma pequena obra prima [14]. Ele usa o sistema de referência de momento infinito e o limite de grande momento e energia transferidos para chegar ao comportamento de escala na variável $\mathrm{x}_{\mathrm{Bj}}=-\mathrm{q}^{2} / \mathrm{M} \nu$ com $\mathrm{M}$ a massa do núcleon, $\mathrm{q}^{2}$ o quadrado do quadrimomento transferido pela corrente eletromagnética ou fraca e $\nu$ a energia transferida.
} 
Bjorken ao voltar a Stanford encontra-se com Feynman graças à iniciativa do seu jovem pós-doutor grego, Emannuel Paschos, que havia estado com Feynman naquela tarde e discutem a interpretação do comportamento de escala de Bjorken em termos dos pártons de Feynman. Por que razão Feynman não publica nada sobre pártons no contexto do comportamento de escala observado experimentalmente no espalhamento inelástico profundo lépton-núcleon? Bjorken e Paschos desenvolvem a ideia de identificar os pártons com os quarks de Gell-Mann e Zweig e analisam os dados experimentais, ainda frescos, com este modelo. Terminam este trabalho em outubro do mesmo ano e Bjorken, antes de publicá-lo, elegantemente espera pelos resultados da análise de Feynman quando este então diz a Bjorken que não espere e publique seu trabalho com Paschos [15]. De fato, o trabalho de Bjorken e Paschos é a primeira publicação a usar o nome párton e embora mencionem Feynman constantemente como sendo o grande motivador dessas ideias, não podem associar Feynman e os seus pártons a nenhuma publicação, já que o artigo deste no Physical Review Letters [9] aparece em dezembro de 1969, 3 meses depois de Bjorken e Paschos!

Podemos agora olhar que ideias Feynman apresenta no seu artigo sobre pártons (ref [9]). Este artigo é extraordinário mesmo para os padrões de Feynman. Nele não encontramos uma única equação embora o texto contenha definições de variáveis utilizadas para descrever os processos físicos que Feynman estuda neste trabalho. Uma delas passará a ser conhecida como variável de escala de Feynman, para distingui-la da variável de escala de Bjorken, mencionada acima. Entre as muitas ideias originais apresentadas neste trabalho, Feynman propõe, pela primeira vez, a distinção entre experimentos exclusivos e inclusivos na análise das colisões hadrônicas. Num experimento exclusivo, o experimental estuda processos em que apenas certas partículas sejam produzidas e não outras (por exemplo, a colisão próton + próton $\rightarrow$ próton + nêutron + píon $^{+}$), enquanto num experimento inclusivo busca-se estudar o comportamento de uma determinada partícula no estado final, em um certo intervalo cinemático de momento longitudinal e transversal, não importando que outras partículas hajam sido produzidas juntamente com ela, por exemplo: próton + próton $\rightarrow$ pion $^{+}+\mathrm{X}$ onde o estado final $\mathrm{X}$ não é especificado. Feynman sugere neste artigo que o comportamento a altas energias das seções de choque inclusivas deve apresentar um comportamento de escala quando a energia total for muito grande e o momento transversal da partícula no estado final (no exemplo acima o píon ${ }^{+}$) for limitado. Neste caso toda a dependência da seção de choque nas variáveis cinemáticas do píon expressase como uma função da variável de escala $\mathrm{x}_{\mathrm{F}}=\mathrm{P}_{\mathrm{z}} / \mathrm{W}$ onde $\mathrm{P}_{\mathrm{z}}$ é o momento longitudinal e $\mathrm{W}$ a energia total da colisão.

Feynman, sendo antes de mais nada um teórico de campos, vê os pártons como sendo as partículas nuas (bare particles) de uma TQC subjacente, uma ideia que sequer é exposta neste artigo, sendo encontrada na referência [10], sua longa contribuição numa conferência em Stony Brook em setembro de 1969. Para chegar aos pártons, Feynman, em comum com Bjorken [14], usa o sistema de referência de momento infinito pois neste sistema o próton é contraído na forma de uma fina panqueca e a interação com a sonda, seja ela eletromagnética como no caso do espalhamento de elétrons, ou fraca, no espalhamento de neutrinos, ou forte, na colisão entre dois hádrons, é instantânea e assim sendo o estado interno dos hádrons é congelado e a interação entre os pártons pode ser ignorada podendo ser então tratados como partículas livres durante a colisão. Esta é a essência do modelo a pártons 5

\section{Conclusões}

O nascimento do modelo a pártons ilustra de maneira exemplar a fertilidade do pensamento de Feynman e sua capacidade de perseguir uma ideia simples e aplicá-la com sucesso na descrição de fenômenos complexos que estejam sob investigação experimental. Este episódio mostra também o quão saudável é para o desenvolvimento científico a ausência de escolas de pensamento dominantes e a coexistência de várias linhas de investigação. Neste caso foi fundamental que uma parte da comunidade buscasse resgatar, através da álgebra de correntes, uma desacreditada TQC para as interações fortes (Bjorken, Gell-Mann, entre muitos outros. Ver Tao [16] para uma excelente análise deste período).

O modelo a pártons converteu-se numa ferramenta indispensável para a a análise e interpretação dos dados obtidos em experimentos de altas energias [18]. O comportamento de escala observado inicialmente nos dados experimentais criou uma situação paradoxal sugerindo uma teoria quântica de campos livres mas que ao mesmo tempo deveria impedir a presença de quarks no estado final das colisões. A solução deste paradoxo deu-se com a formulação da Cromodinâmica Quântica, uma teoria que se comporta assintoticamente livre (altos momentos transferidos sondam pequenas dimensões espaço-temporais) mas que a baixas energias (largas distâncias) confina seus quanta impedindo sua aparição como estados livres (consultar Tao [16] para a história do surgimento da Cromodinâmica Quântica).

Terminamos este artigo com duas curiosidades sobre o Physical Review Letters de Feynman [9], sempre citado quando se fala sobre os pártons. A primeira curiosidade é que neste artigo a palavra pártons não aparece nenhuma vez e a segunda é que este é o artigo publicado por Feynman que mais citações recebeu, segundo a base de

\footnotetext{
${ }^{5}$ Feynman publica sua análise dos experimentos de espalhamento inelástico profundo usando o modelo a pártons em um livro que se tornou um clássico ("Photon-Hadron Interactions" [17]), baseado no curso avançado de pós-graduação que ele ministrou no Caltech no ano acadêmico 1971-72.
} 
dados INSPIRE, usada em Física de Altas Energias. Mais mesmo que seus trabalhos sobre eletrodinâmica quântica ou integral de caminho, num total de 2123 citações!

\section{Agradecimentos}

Agradeço Nelson Studart por convidar-me a participar deste volume celebrando o centenário de Richard Feynman.

O Fermilab é operado pela Fermi Research Alliance, LLC sob o contrato número DE-AC02-07CH11359 com o Departamento de Energias dos EUA.

\section{Referências}

[1] R.P. Feynman e M. Gell-Mann, Phys. Rev. 109, 193 (1958).

[2] R. Feynman, F. Morinigo, W. Wagner, B, Hatfield e D. Pines, in Feynman Lectures on Gravitation editado por F.B. Moringo, W.G. Wagner e B. Hatfield (AddisonWesley, Boston, 1995).

[3] R.P. Feynman e G. Speisman, Phys. Rev. 94, 500 (1954).

[4] G.F. Chew e S.C. Frautschi, Phys. Rev. Lett. 8, 394 (1961).

[5] H. Pietschmann, ArXiv:1101.2748 (2011).

[6] M. Gell-Mann, Phys. Rev. 125, 1067 (1962).

[7] M. Gell-Mann, Phys. Lett. 8, 214 (1964).

[8] G. Zweig, CERN-TH-401 (1964).

[9] R.P. Feynman, Phys. Rev. Lett. 23, 1415 (1969).

[10] R.P. Feynman, in High Energy Collisions editado por C.N. Yang (Gordon and Breach, New York, 1969).

[11] M. Riordan, The Hunting of the Quark (Simon and Schuster, New York, 1987).

[12] J.I. Friedman, Rev. Mod. Phys. 63, 615 (1991).

[13] F. Close, The Infinity Puzzle (Oxford University Press, Oxford, 2011).

[14] J.D. Bjorken, Phys. Rev. 179, 1547 (1969).

[15] J.D. Bjorken e E.A. Paschos, Phys. Rev. 185, 1975 (1969).

[16] T.Y. Tao, From Current Algebra to Quantum Chromodynamics (Cambridge University Press, Cambridge, 2010).

[17] R.P. Feynman, Photon-Hadron Interactions (AddisonWesley, Boston, 1972).

[18] J. Campbell, J. Huston e F. Krauss, The Black Book of Quantum Chromodynamics, a Primer for the QCD Era (Oxford University Press, Oxford, 2017). 